\title{
The effect of curb parking on road capacity and traffic safety
}

\author{
Y. Cao ${ }^{1} \cdot$ Z. Z. Yang $^{2} \cdot$ Z. Y. Zuo ${ }^{1}$
}

Received: 25 February 2016 / Accepted: 13 December 2016 / Published online: 29 December 2016

(C) The Author(s) 2016. This article is published with open access at SpringerLink.com

\begin{abstract}
Purpose In order to explore the effect of curb parking on dynamic traffic and formulate some reasonable and effective traffic management measures, the effects on capacity and traffic safety are studied.

Methods Four road segments with curb parking were selected as the investigation location. Several traffic characteristics and parameters were then investigated and counted, including traffic flow volume, speed, headway and traffic conflict. Based on a reduction of lane effective width and the Gap Acceptance Theory, two effect models describing curb parking and road capacity were constructed. Considering the factors involved in motor vehicle conflicts (such as non-motor vehicle lane distribution and stopping sight distance), the effects of curb parking on both motor vehicle and non-motor vehicle traffic safety was analyzed, as well as pedestrian crossing safety. This case study analyzes both the capacity and traffic safety of all four investigation segments.

Results Results show that the lane effective width of road segment with curb parking significantly influences the road capacity and impacts the traffic flow volumes of the parking lane and its adjacent lane.
\end{abstract}

Y. Cao

caoyi820619@aliyun.com

Z. Z. Yang

yangzhongzhen@263.net

Z. Y. Zuo

zuozy@djtu.edu.cn

1 School of Transportation Engineering, Dalian Jiaotong University, Huanghe Rd. 794, 116028 Dalian, People's Republic of China

2 Transportation Management College, Dalian Maritime University, Linghai Rd. 1, 116026 Dalian, People's Republic of China
Conclusions Curb parking significantly effects traffic operation safety; however, it can be improved by implementing traffic safety management measures.

Keywords Traffic engineering · Curb parking · Capacity . Traffic safety $\cdot$ Conflict $\cdot$ Gap acceptance theory

\section{Introduction}

As a result of growing automobile population, cities frequently permit parking or temporary parking in road segments without heavy traffic. In order to reduce the occupancy of curb parking in road resources, the management mode of parallel curb parking is not only widely used in China but also in most other countries with parking meters (see Fig. 1). Practice shows that curb parking can relieve the impact of insufficient off-street parking facilities to some extent. It also has a high convenience factor, as Geng's study reveals [1]. However, some scholars believe that curb parking has a significant impact on urban dynamic traffic, as suggested in Dai's study [2]. Therefore, it is necessary to study the main influence of curb parking quantitatively using actual traffic investigation data. The results from this present study can provide a theoretical reference for the formulation and implementation of parking management measures.

Both domestic and foreign scholars have already begun to study curb parking and its impact. Based on the results of a parallel and oblique curb parking investigation, Yousif [3] discussed the traffic operation status in curb parking road segments by analyzing the parking time and acceptance gap. Jaller [4] studied the problem of parking heavy trucks in a city. He proposed an estimation method of curb parking demand. By investigating the transverse occupying width of curb parking, Furth [5] analyzed the distribution regularities 
Fig. 1 The application of curb parking in China and other countries
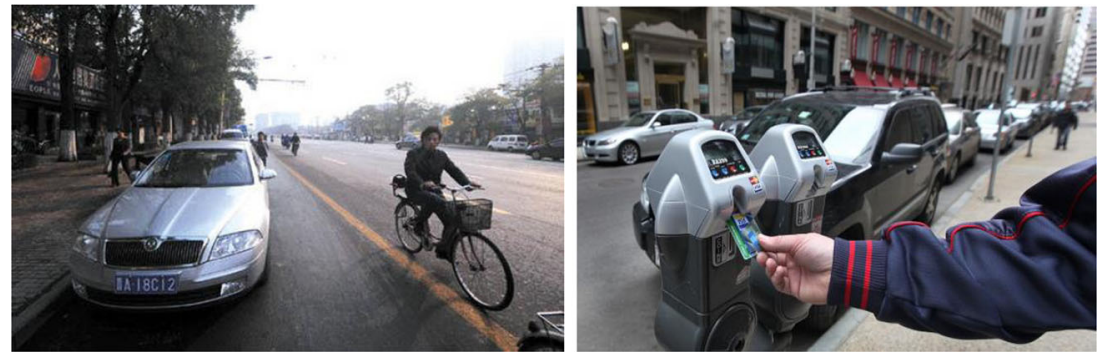

of the effective lane width using a statistical method. In addition, he also studied the influence of curb parking on the travelling space of non-motorized vehicles. Through a driving simulation experiment, Edquist [6] studied the influence of curb parking on a vehicle's travelling speed and the driver's reaction time by considering the driver's psychological characteristics. Wijayaratna [7] developed a mathematical model for calculating a "capacity adjustment factor" which quantifies the impact of on street parking on the capacity of an arterial road. This factor was applied to estimate the capacity of selected arterial roads in Sydney, Australia. The case study presented a reduction in capacity of up to $17 \%$ for short time restriction parking zones present on arterial roads.

Here in China, He [8] acquired the modification coefficient of road capacity under this condition by analyzing the remaining quantity of lanes, width of lane and transverse width under curb parking conditions. Based on several kinds of parking behaviors, Liu [9] not only constructed the cellular automation traffic flow model, but also analyzed the time-space relationship of curb parking. Using the method of life time analysis method, Guo [10] constructed a model of the relationship between crossing and the volume of nonmotorized vehicle specifically focusing on the behavior of non-motorized vehicles occupying motor vehicle lanes with curb parking. He also analyzed the influence of curb parking on the traffic behavior of non-motorized vehicles. For the two kinds of vehicles arriving at a discrete flow and continuous flow, Mei [11] constructed and verified the delay model and the following model, respectively, under the influence of curb parking. Mei [12] also constructed an effective model showing the impact of curb parking on motor vehicle travelling speed under a mixed traffic condition. Based on the airflow analysis theory of hydromechanics and considering the non-motorized vehicle travelling and compression characteristic, Chen [13] constructed the traffic wave model to explain non-motorized vehicle compression influenced by curb parking.

Although several studies on this topic have been carried out both here and abroad in general, most of the studies abroad focused their attention on the characteristics of curb parking itself rather than its influence on dynamic traffic. In China, scholars focused their attention on the study of its influence on capacity, in respect to the reduction of effective lane width rather than the type and amount of traffic conflicts caused by curb parking. Additionally, the influence of curb parking on the safety of dynamic traffic should be intensified furthermore. Given such a status, this paper discusses the calculation method of capacity in road segments with curb parking according to an actual traffic investigation and considering the two factors of effective lane width and acceptable inserting gap. The influence of curb parking on traffic safety is also analyzed in this paper by considering motor vehicle conflict, stopping sight distance, and the transverse distribution of nonmotorized vehicles. Significant theoretical and practical value can be found in formulating and implementing traffic management measures on curb parking street.

\section{Traffic investigation and data processing}

\subsection{The scheme of traffic survey}

Shenban Rd., Dengyun Rd., Shangtang Rd. and Xiangjisi Rd. of Hangzhou were the four road segments selected as the investigation segments in this research. The four segments are secondary or branch roads. The traffic states on them are not congested at peak hours. In order to relieve the problem of insufficient off-street parking facilities, parallel curb parking facilities are set in all of these segments, making these segments appropriate for this research.

Practical experience shows that, when the traffic volume is larger, the influence of curb parking on dynamic traffic should be more significant. So this traffic investigation should be conducted during the peak morning or evening hours, which is 7:00-9:00 and 16:00-18:00 every day. The investigation was conducted from Tuesday to Thursday.

The investigation was divided into two stages: external observation and internal arrangement. In external observation the dynamic traffic operating state and parking state are recorded by video observation. The vehicle's speed can be measured and recorded by portable radar velocimeter while the occupying width of different parking can be recorded by manual measurement. In internal arrangement, according to the video record, traffic data can be acquired, including: traffic volume, headway and the type and quantity of traffic conflict. 
Table 1 Geometric structure of the investigation road segments

\begin{tabular}{lllll}
\hline Road segments & $\begin{array}{l}\text { Mount of lanes in } \\
\text { one-way }\end{array}$ & $\begin{array}{l}\text { Width of outside } \\
\text { motor lane }(\mathrm{m})\end{array}$ & $\begin{array}{l}\text { Width of non-motor } \\
\text { lane(m) }\end{array}$ & $\begin{array}{l}\text { Average occupying } \\
\text { width }(\mathrm{m})\end{array}$ \\
\hline Shenban Rd. & 1 & 3.2 & 2.0 & 1.92 \\
Dengyuan Rd. & 1 & 4.6 & 0.0 & 1.90 \\
Shangtang Rd. & 3 & 3.5 & 3.0 & 2.24 \\
Xiangjisi Rd. & 3 & 3.5 & 2.5 & 2.05 \\
\hline
\end{tabular}

\subsection{The investigation location}

The geometric features of the investigation segments are shown in Table 1, according to the parking occupying width results of the investigation.

\subsection{Traffic data acquisition}

Several kinds of data can be acquired by manually counting observations from the video record, such as traffic volume, quantity of curb parking and quantity of traffic conflicts. By sampling the observation with a radar velocimeter, the travelling speed of vehicles can also be acquired. The investigation data from 8:00-9:00 of the first day is listed as an example in Table 2.

The traffic parameters acquired in this investigation can be used to calibrate the calculation parameters of a capacity model under curb parking conditions. The parameters can also be used to analyze the influence of curb parking on dynamic traffic safety quantitatively.

\section{The influence of curb parking on road capacity}

\subsection{For the segments without a non-motor lane}

For this situation, the capacity of a road segment with curb parking should be affected significantly in the parking lane and its medial adjacent lane. Accordingly, the analysis and calculation of capacity should focus on the two kinds of lanes mentioned above. For the other lanes, the influence should be far less than on the two lanes mentioned above. Experience shows that the transverse space of a road will be occupied by curb parking. However, the transverse remaining width with curb parking will affect the travelling situation of dynamic traffic directly. Thus, the influence of curb parking on capacity should be divided into two situations and discussed according to the transverse remaining width.

1. The reduction of effective lane width

The distance from the inner lane line of the medial lane adjacent to the parking zone to the inner edge of the curb parking zone is defined as the transverse remaining width and is marked as $W_{S}$. Traffic investigation shows that when $W_{S}$ is larger than a certain value the transverse remaining space of the curb parking segment can be used as two lanes, as shown in Fig. 2. According to previous research [14], by considering the transverse safety distance of travelling vehicles and the vehicle's standard width, the critical value of the transverse remaining width $W_{S}$ can be calculated. These values for low speed situations are listed in Table 3.

In Table 3, the standard width of both the car and truck is a statistical parameter, which is appropriate for a huge majority of modern cars and trucks. According to the analysis above, when the transverse remaining width $W_{S}$ is larger than the critical value listed in Table 3, the reduction of the lane's effective width is the main reason why the capacity of the lane is reduced. Because of this, the modified method about lane width mentioned in Highway Capacity Manual (HCM 2010) [15] can be used to modify the capacity of a lane under this situation, as shown by Eq. (1) and Eq. (2).

$C_{l 1}=C_{l 2}=C_{0} \cdot f_{w}$

$f_{w}=1+\frac{\left(0.5 \cdot W_{S}-W\right)_{c}}{9.144}$

Where.

$C_{l 1}$ and $C_{l 2}$ the capacity of lane 1 and lane 2, as Fig. 2 shows $(\mathrm{pcu} / \mathrm{h})$,
Table 2 The traffic data in the $1 \mathrm{st}$ investigation day (8:00-9:00)

\begin{tabular}{lllll}
\hline Road segments & Traffic volume (pcu/h) & Average speed $(\mathrm{km} / \mathrm{h})$ & $\begin{array}{l}\text { The max quantity } \\
\text { of parking }\end{array}$ & $\begin{array}{l}\text { The quantity } \\
\text { of traffic conflicts }\end{array}$ \\
\hline Shenban Rd. & 243 & 40.79 & 5 & 8 \\
Dengyuan Rd. & 444 & 44.40 & 3 & 6 \\
Shangtang Rd. & 495 & 45.64 & 3 & 14 \\
Xiangjisi Rd. & 792 & 48.26 & 9 & 39 \\
\hline
\end{tabular}


Fig. 2 The transverse remaining width and lane sharing

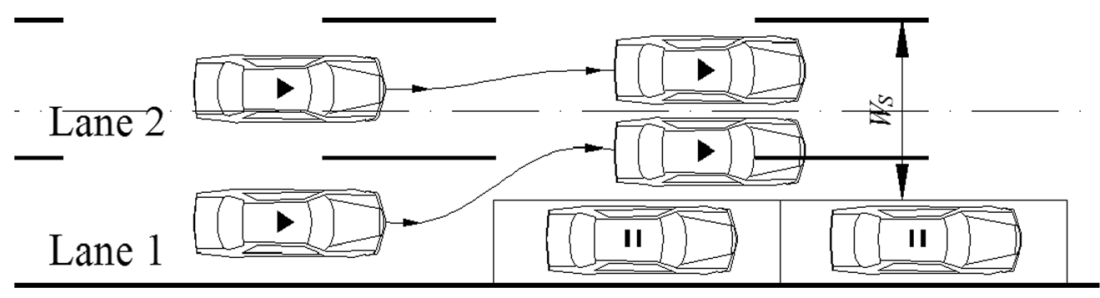

$C_{0}$ the basic capacity of one lane (pcu/h),

$f_{w}$ the correction coefficient of lane width.

$W_{c}$ the standard lane width in certain country. Its value is $3.66 \mathrm{~m}(12 \mathrm{ft})$ for USA, $3.75 \mathrm{~m}$ in China.

In Eq. (2), the 9.144 is caused by unit conversion from English to metric unit. Further analysis indicates that, although two vehicles can travel abreast within this transverse remaining width for the situation above, the lateral clearance for vehicle travelling is insufficient. In addition, because the medial lane line of parking zone loses the traffic function, there will be a very adverse effect on traffic safety.

\section{The Gap Acceptance model}

When the transverse remaining width is less than the critical value listed in Table 3, mainline traffic flow cannot travel parallel. Thus, vehicles in the parking lane will be forced to change lane and travel using the acceptable gap of the fleet in its medial adjacent lane, as shown in Fig. 3. For this situation, there will be a serious bottleneck of capacity in the road segment with curb parking. This is because a large number of motor vehicles in lane 1 are forced to combine travel using the acceptable gap. This kind of traffic operation condition will lead to a large number of traffic conflicts and the capacity of lane 2 will also be affected.

The headway of the fleet in lane $2 h(\mathrm{~s})$ is assumed to follow negative exponential distribution with parameter $\lambda$. Then, the distribution function of $h$ is shown as Eq. (3).

$F(t)=P(h \leq t)=1-e^{-\lambda t}$

If $t_{0}(\mathrm{~s})$ is the critical time interval of the fleet in lane 2 , it means the minimum headway of this fleet for vehicles travelling through this fleet. $t(\mathrm{~s})$ is the car-following headway in lane 1 for successive travelling through the fleet in lane 2 . There are $n$ vehicles in lane 1 waiting for driving into lane 2 . Then, the probability of the headway in lane 2 which allows $n$ vehicles to driving into it fitly can be expressed by Eq. (4).

Table 3 The critical value of transverse residual width $W_{S}$

\begin{tabular}{clllll}
\hline $\begin{array}{c}\text { Vehicle type of the } \\
\text { parking lane }\end{array}$ & $\begin{array}{c}\text { Standard } \\
\text { width }(\mathrm{m})\end{array}$ & \multicolumn{4}{l}{ Travelling speed $(\mathrm{km} / \mathrm{h})$} \\
\cline { 3 - 6 } & & 10 & 20 & 30 & 40 \\
\hline Car & 2.0 & 5.3 & 5.5 & 5.7 & 5.9 \\
Truck & 2.55 & 5.7 & 5.9 & 6.1 & 6.3 \\
\hline
\end{tabular}

$P(n)=P\left[t_{0}+(n-1) t \leq h<t_{0}+n t\right]=e^{-\lambda t_{0}}\left[e^{-\lambda(n-1) t}-e^{-\lambda n t}\right]$

When the headway is longer than $t_{0}+n t$, there are also $n$ vehicles can travel into lane 2 . Because there are only $n$ vehicles in lane 1 . So, this probability can be marked as $P^{\prime}(n)$, as Eq. (5) shown.

$P^{\prime}(n)=P\left[h>t_{0}+n t\right]=1-P\left[h \leq t_{0}+n t\right]=e^{-\lambda t_{0}} e^{-\lambda n t}$

Given the traffic volume $q_{l 2}$, that is also the total number of headways in lane 2 in one hour. Thus, the number of vehicles in lane 1 can travel into lane 2 can be marked as $Q$. It can be calculated by Eq. (6).

$Q=q_{l 2} \sum[P(n) \cdot n]+q_{l 2} P^{\prime}(n) \cdot n=q_{l 2} e^{-\lambda t_{0}} \frac{1-e^{-\lambda n t}}{1-e^{-\lambda t}}$

According to the definition of capacity, the number of vehicles in lane 1 should be assumed to approach infinity. So, the capacity of lane 2 of the segment with curb parking $C^{\prime}{ }_{12}(\mathrm{pcu} /$ h) should be expressed as Eq. (7). It also can be plotted as Fig. 4 shown. The meaning of parameter $\lambda(\mathrm{pcu} / \mathrm{s})$ is the average arrival rate of the vehicles in lane 2. It also equals to $q_{12} /$ $3600 \mathrm{~s}$.

$C^{\prime}{ }_{l 2}=q_{l 2} \frac{e^{-\lambda t_{0}}}{1-e^{-\lambda t}}+q_{l 2}$

It can be known from Fig. 4, all the curves of the capacity of lane $2 C^{\prime}{ }_{12}$ decrease first and then increase with different $t_{0}$ and $t$. However, all of them are less than the basic traffic capacity of one lane with the corresponding design speed of the road. By analyzing the model above, it can be shown that the capacity of lane 2 for this traffic condition is mainly determined by the traffic volume of lane 2 , besides the critical acceptance gap in lane 2 and the car-following headway in lane 1. In addition, this effect is bidirectional. That means when the volume in lane 2 is less, the number of vehicles in lane 1 which can travel through lane 2 using an acceptable gap is greater. So the capacity will be compensated. On the contrary, when this volume is larger, only a few vehicles in lane 1 can realize the combined travelling with an acceptance gap. It needs to be added that, The essence of the conflict between the parking vehicle when it leaves and the passing vehicles is also similar to this situation. 
Fig. 3 Combined driving by acceptable gaps

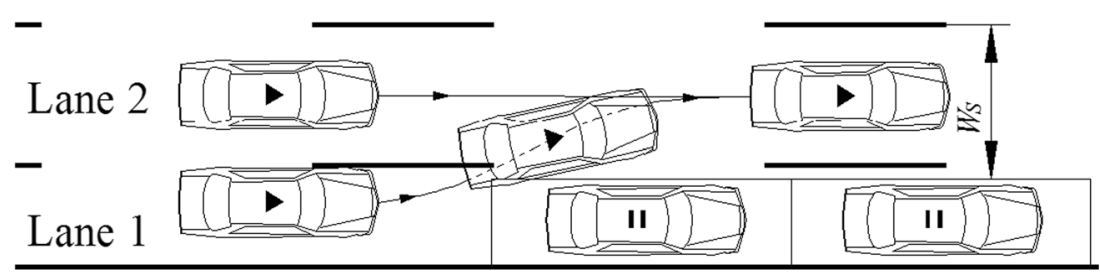

\subsection{For segments with a non-motor lane}

For this situation, there will be no conflicts between motor vehicles. However, some non-motor vehicles will be forced to travel in the medial adjacent motor lane. These non-motor vehicles will occupy the space of the motor lane. This phenomenon will lead to the reduction of the motor lane effective width, which is $W_{E}$, as shown in Fig. 5. Thus, the influence mechanism of curb parking on capacity is similar to the reduction of effective lane width, as discussed above in section 3.1. However, the correction coefficient of the lane width $f_{w}$ should be calculated by Eq. (8).

$f_{w}=1+\frac{\left(W_{E}-W_{C}\right)}{9.144}$

\section{The influence of curb parking on traffic safety}

\subsection{The definition and identification of traffic conflict}

Traffic conflict is defined as the obvious reduction of travelling speed caused by another adjacent vehicle. In actual observation, this conflict can be identified by the variety of vehicle's brake lights. The actual investigation shows that additional traffic conflicts caused by curb parking exist in 3 kinds of situations. The first is the conflict between the curb parking vehicles and the travelling vehicles in the parking lane. The second is the conflict between the mainline traffic and the travelling vehicles in the parking lane when they travel into the medial adjacent lane. The third one is the conflict between the blocked vehicle and its following vehicles, as shown in Fig. 6.

\subsection{The influence on motor vehicle safety}

The influence of curb parking on motor vehicle safety can be analyzed according to the quantity of traffic conflicts caused by it. If the quantity of traffic conflicts is larger, then the hidden danger caused by curb parking is more serious.

Further analysis indicates that the traffic conflicts mentioned above are related to the traffic densities in the parking lane and in the medial adjacent lane. This means that when the densities are larger, the quantity of traffic conflicts should be larger as well. The traffic conflicts in a road segment with curb parking parameter can be acquired for analysis by actual traffic investigation. Using and comparing these data, motor vehicle safety can then be analyzed quantitatively.

\subsection{The influence on non-motor vehicle safety}

The influence of curb parking on non-motor vehicle safety can be analyzed by observing the non-motor vehicle lane distribution characteristic. Observation shows that, because curb parking occupies the road space to different extents, the nonmotor vehicles which should originally travel in the non-
Fig. 4 The capacity of lane $2 C^{\prime}{ }_{22}$ at different conditions

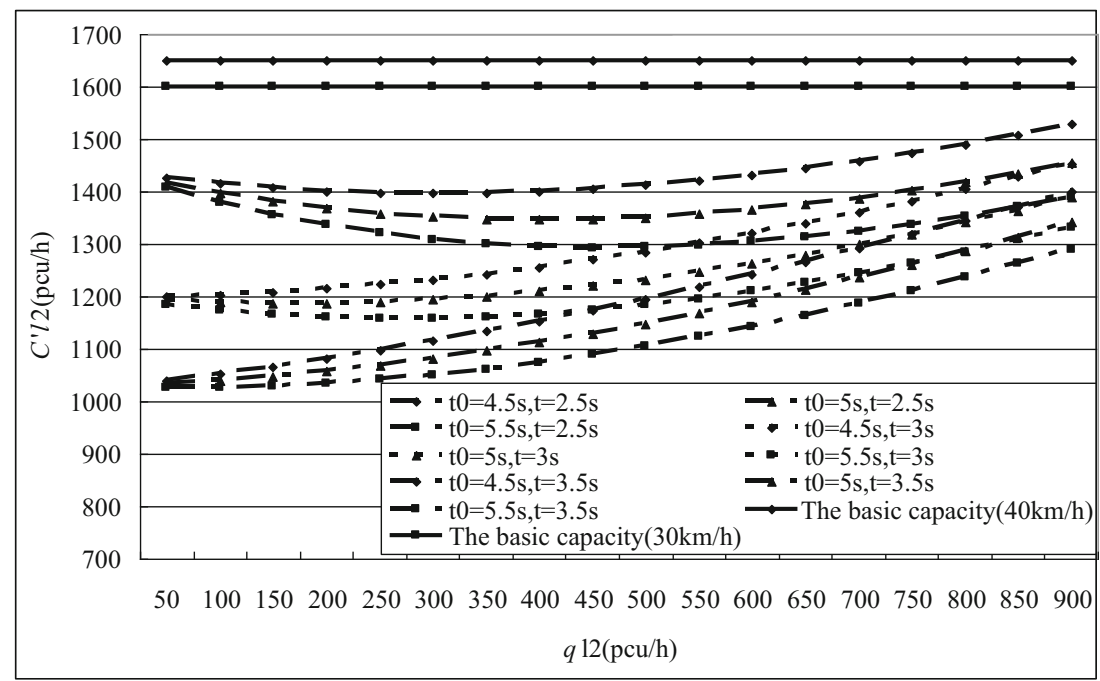


Fig. 5 The influence mechanism of curb parking on capacity for non-motor lane

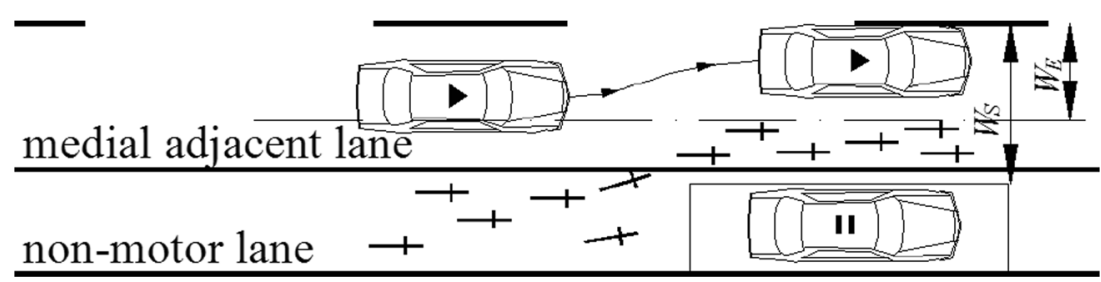

motor vehicle lane or the outside lane are forced to travel into the medial adjacent lane. Thus, a serious interference occurs between mainline traffic and non-motor vehicles. This situation reduces traffic safety to a great extent, as shown in Fig. 5.

According to the safety influence mentioned above, it can be recognized that this kind of influence should be related to some extent to the volume of non-motor vehicles, traffic density in the medial adjacent lane, and the transverse remaining width in road segments with curb parking.

\subsection{The influence on crossing pedestrian safety}

The influence of curb parking on safety of pedestrians crossing the street is caused by parked vehicles blocking a driver's sight line. Accordingly, this influence should be analyzed from in relation to the stopping sight distance, as shown in Fig. 7.

According to this mechanism, the position of the most unfavorable conflict point between motor vehicles and cross-street pedestrians should be determined first. Then, from this conflict point the stopping sight distance $S_{T}(\mathrm{~m})$ and pedestrian sight distance $S_{P}(\mathrm{~m})$ should be measured through the motor vehicle driving direction and pedestrian travelling direction, respectively. Thus, the sight triangle can be constructed. In order to not block the driver's sight line, there should not be any curb parking vehicles in the scope of the sight triangle. The analysis above contributes to designing the curb parking zone more safety and reasonably in road segments with pedestrian crossings.

The stopping sight distance of motor vehicles $S_{T}(\mathrm{~m})$ can be calculated by Eq. (9). Assuming the pedestrian crossing the road on a steady mode with a constant speed, the critical distance of pedestrian $S_{P}(\mathrm{~m})$ can be calculated by Eq. (10). Thus, the safety distance $S(\mathrm{~m})$, which is from the end point of the curb parking zone to the upstream edge of the pedestrian crosswalk can be calculated by the geometrical relationship of the sight triangle, as shown in Eq. (11).

$S_{T}=\frac{V_{T}}{3.6} \cdot t_{r}+\frac{V_{T}^{2}}{254\left(\varphi_{T}+f+i\right)}$

$S_{P}=2 \cdot V_{P} \frac{S_{T}}{V_{T}}$

$S=\frac{S_{T}\left(W-L+S_{P}\right)}{S_{P}}=\frac{V_{T}\left(W-L+S_{P}\right)}{2 \cdot V_{P}}$

Where.

$V_{T}$ the average travel speed of vehicles on the segment $(\mathrm{km} / \mathrm{h})$. $t_{r}$ the reaction time of driver and machine of vehicle(s). For security reasons, it should be $2.5 \mathrm{~s}$.

$\varphi_{T}$ the coefficient of friction between vehicle's tire and asphalt pavement. According to the principles of conservative design, the adverse condition of wet road surface should be considered. So, its value should be 0.3 .

$f$ the coefficient of rolling resistance of vehicle's tire. 0.02 is normal value.

$i$ the slope through the direction of pedestrian crossing street. If upslope, it is positive (\%).

$V_{P}$ the travel speed of crossing pedestrian $(\mathrm{km} / \mathrm{h})$. It is $5 \mathrm{~km} /$ $\mathrm{h}$ for the most unfavorable situation.

$W$ the width of parking zone(m). It should be measured at actual location.

$L$ the distance from the most unfavorable conflict point to the road's edge $(\mathrm{m})$.

For example, if $W$ is $2.5 \mathrm{~m}, L$ is $4 \mathrm{~m}$, for the horizontal road, the safety setting distance $S(\mathrm{~m})$ from the end point of the curb parking zone to the upstream edge of the pedestrian crosswalk can be calculated as Table 4 . at different average travel speed.
Fig. 6 The identification and types of traffic conflict

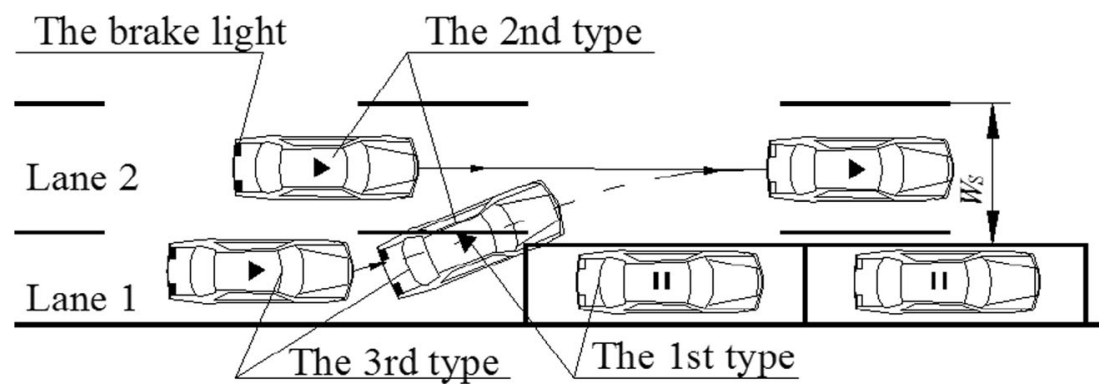


Fig. 7 The safety effect of curb parking on crossing pedestrian

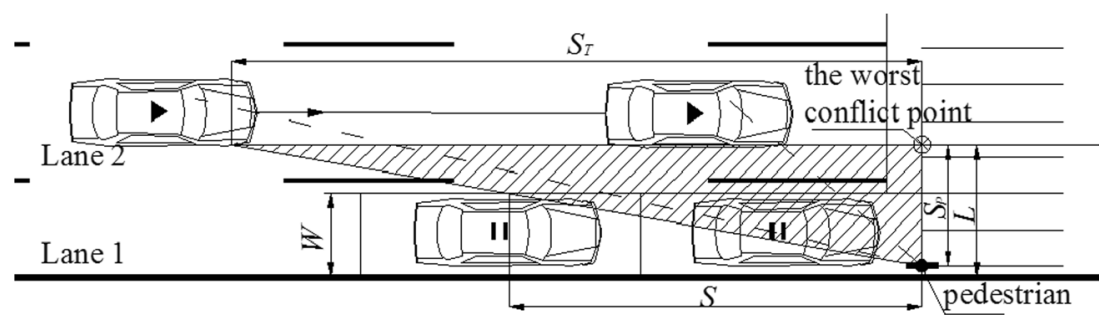

It can be known from Table 4, the safety setting distance $S(\mathrm{~m})$ from parking zone to pedestrian crosswalk is determined by the average travel speed $V_{t}$ to a great extent. Its value is larger than the current standard of $5 \mathrm{~m}$ in Europe. So, this setting criterion of parking zone is more specific and secure than current criterion in Europe.

\section{The case study}

According to the traffic data acquired from the four investigated road segments, an analysis of the capacity and traffic safety under the influence of curb parking can be conducted.

\subsection{Capacity analysis}

\section{(1) Selection of calculation method}

The transverse remaining width $W_{S}$ of road segments with curb parking can be calculated according to the geometric structure data of the investigation road segments listed in Table 1. By comparing these results with the data in Table 3, the calculation method of road segment capacity can be selected. The calculation results of transverse remaining width are listed in Table 5. The average speed in Table 5 is the average value from 7:00 to 9:00 and from 16:00 to 18:00 in the first day.

From the calculation results listed in Table 5, all of the lane transverse residual widths $W_{S}$ of the 4 segments are less than its critical width. This means that the vehicles in the parking lane can travel through the segment by the acceptable gap of the fleet in its medial adjacent lane. Thus, the capacity should

Table 4 The safety setting distance $S(\mathrm{~m})$ from parking zone to pedestrian crosswalk

\begin{tabular}{llll}
\hline $\begin{array}{l}\text { The average } \\
\text { travel speed } \\
V_{t}(\mathrm{~km} / \mathrm{h})\end{array}$ & $\begin{array}{l}\text { The stopping } \\
\text { sight distance } \\
S_{t}(\mathrm{~m})\end{array}$ & $\begin{array}{l}\text { The critical } \\
\text { distance } S_{p}(\mathrm{~m})\end{array}$ & $\begin{array}{l}\text { The safety setting } \\
\text { distance } S(\mathrm{~m})\end{array}$ \\
\hline 20 & 18.81 & 9.41 & 15.8 \\
30 & 31.91 & 10.64 & 27.4 \\
40 & 47.46 & 11.87 & 41.5 \\
\hline
\end{tabular}

be calculated by the acceptable gap formula. Additionally, the lane residual width of Dengyun $\mathrm{Rd}$. is only $2.7 \mathrm{~m}$, which is less than the standard width of one lane. As a result, it is necessary to reduce its capacity according to its lane width after the capacity calculation.

\section{(2) Calculation result and analysis}

The traffic volume data in the medial lane adjacent to parking zone in period of 7:00-9:00 and 16:00-18:00 on the first day were selected as the calculation parameters. Using Eq. (7), the capacity of each medial lane adjacent to its respective parking zone is calculated. For calculation, the critical time interval of the fleet in the medial adjacent lane $t_{0}$ is selected as $4.5 \mathrm{~s}$. The car-following headway for successive travelling through the fleet $t$ is selected as $2.5 \mathrm{~s}$. The calculated capacities of the medial lanes adjacent to curb parking zones and the hourly traffic volume of this lane for all four road segments are shown in Fig. 8.

In Fig. 8, the histograms mean the traffic volume in the medial lane adjacent to parking lane of the 4 road segments. The line plots mean the capacity of the medial lane of these road segments. From Fig. 8, we can see that the capacities at each lane are small. Among them, the capacities of the medial lane adjacent to parking zone in Shenban Rd., Shangtang Rd., and Xiangjisi Rd. are all about $1400 \mathrm{pcu} / \mathrm{h}$. However, because of the influence of the lane width reduction, the capacity of that lane in the Dengyun Rd. segment is about $1240 \mathrm{pcu} / \mathrm{h}$. Compared with the capacities of one ordinary lane for $30 \mathrm{~km} / \mathrm{h}$ and $40 \mathrm{~km} / \mathrm{h}$ design speed without curb parking, these calculation results are all less than the standard value of $1600 \mathrm{pcu} / \mathrm{h}$ (for $30 \mathrm{~km} / \mathrm{h}$ ) and $1650 \mathrm{pcu} / \mathrm{h}$ (for $40 \mathrm{~km} / \mathrm{h}$ ). Thus, the capacity values calculated by the model are less about $12 \%$ (for only combined driving) or $22 \%$ (for both combined driving and reduction of lane width) than the basic capacity of one lane. This decrement is caused by curb parking. According to the analysis above, the influence of the traffic volume in this lane on its capacity is minimal; however, the influence of curb parking itself is significant.

\subsection{Traffic safety analysis}

The operational safety of motor vehicles can be estimated using the quantity of traffic conflicts. The quantity of traffic 
Table 5 The calculation result of transverse residual width $W_{S}$

\begin{tabular}{lllll}
\hline Road name & $\begin{array}{l}\text { Remaining } \\
\text { width } W_{S}(\mathrm{~m})\end{array}$ & $\begin{array}{l}\text { Average } \\
\text { speed }(\mathrm{km} / \mathrm{h})\end{array}$ & $\begin{array}{l}\text { Critical width } \\
\text { of } W_{S}(\mathrm{~m})\end{array}$ & Calculation method \\
\hline Shenban Rd. & 3.28 & 32.5 & 5.7 & Acceptance gap \\
Dengyun Rd. & 2.70 & 31.2 & 5.7 & Acceptance gap \\
Shangtang Rd. & 4.26 & 39.4 & 5.9 & Acceptance gap \\
Xiangjisi Rd. & 3.95 & 42.6 & 5.9 & Acceptance gap \\
\hline
\end{tabular}

conflicts in the parking lane and the medial lane adjacent to it for each of the four segments can be acquired using video data observed on the first day. At the same time, an interval of $30 \mathrm{~m}$ in every segment is selected in the investigated video. The example of Shangtang Rd. is shown in Fig. 9. Thus, how many vehicles are there in this interval in the parking lane and its medial adjacent lane at every 6 min can be counted by playing the video. Then, the average number of vehicles in the interval in each hour can be calculated. At last, the average traffic density of the lanes mentioned above can be acquired by converting this average number form $30 \mathrm{~m}$ to $1 \mathrm{~km}$. The traffic density and conflicts in the parking lane and its medial adjacent lane is shown in Fig. 10.

In Fig. 10, the histograms mean the average traffic density in the parking lane and its adjacent lane of the 4 road segments. The line plots mean the quantity of conflicts in these lanes. From Fig. 10, it can be recognized that the quantity of traffic conflicts which happen in the parking lane and its medial adjacent lane is significantly affected by curb parking. Generally, the traffic density of curb parking road segment is lower then conventional road. If the traffic density of this kind of road segment is higher frequently, the curb parking should not be allowed. Moreover, a relationship between the quantity of conflicts and the traffic densities of the two lanes above can be seen: for the situation of low traffic density, just like this case, when the traffic density is larger, the quantity of traffic conflicts in the lane increases. In addition, when the density is larger, the speed of conflict increasing is fester as well, resulting in poorer traffic safety.

In the four segments observed in this investigation, nonmotor vehicle volume is small. There are several motorcycles or bicycles in the outside lane of the Shangtang Rd. and Xiangjisi Rd. segments. From the video data, the influence of curb parking on the safety of non-motor vehicles is mainly determined by the transverse remaining width after occupying. Because there is only one lane for each direction in the Shenban Rd. and Dengyun Rd. segments, the remaining width after parking only supports one line of motor vehicle travelling. Thus, non-motor vehicles are forced to travel into the motor vehicle lane, increasing the potential safety hazard. However, with the larger transverse remaining width in the Shangtang Rd. and Xiangjisi Rd. segments, non-motor vehicles can travel in the remaining width, which is safer than the other two roads.

In the four segments included in this study, there were no pedestrian crosswalks. However, a few observations of pedestrians going across the road anyways reduced the traffic safety to some extent.
Fig. 8 The traffic volume and capacity of the medial lane adjacent to parking lane

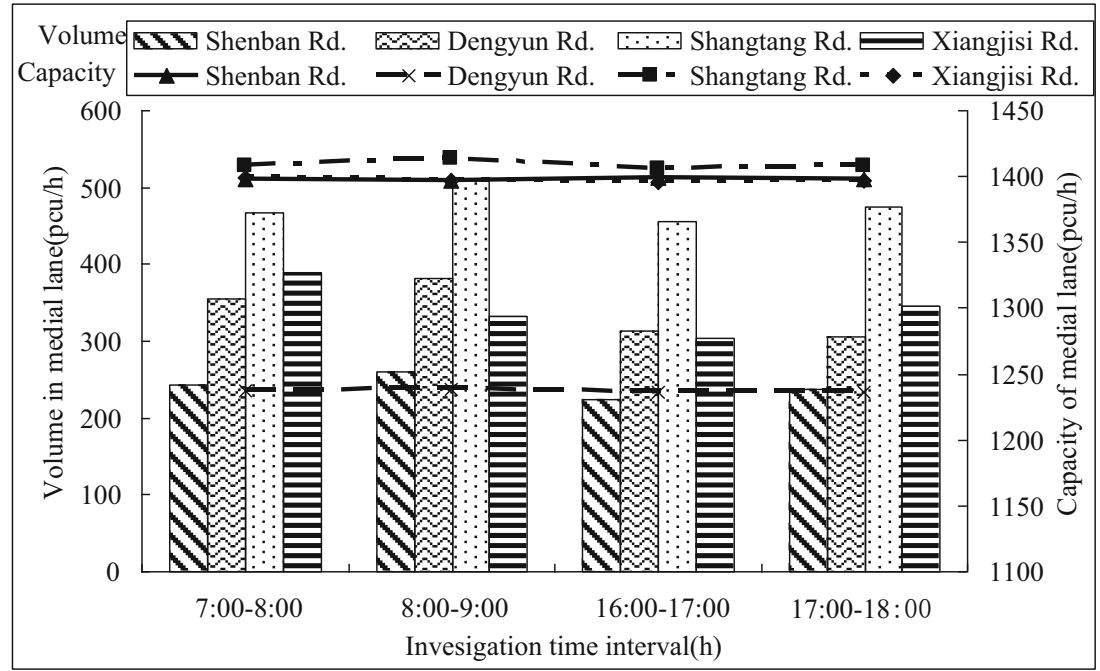




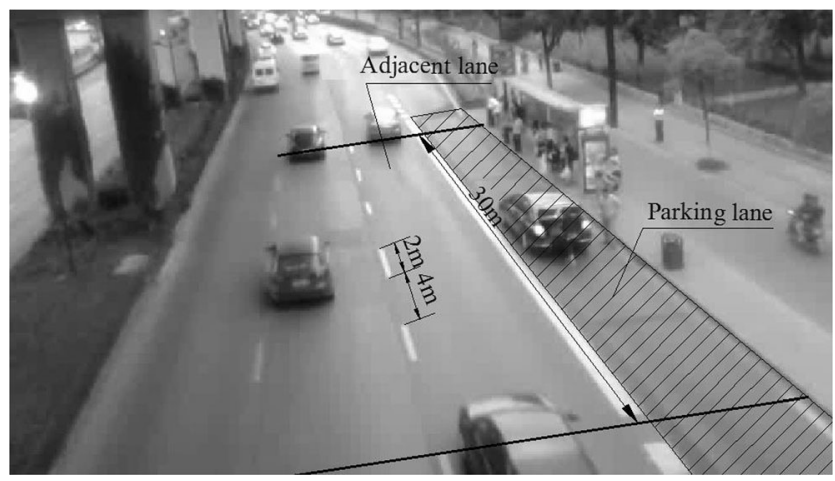

Fig. 9 The measuring method of traffic density

\section{Improvement measures}

Through this theoretical study and case analysis some improvements to curb parking can be summarized in terms of road capacity and traffic safety.

(1) Road segments with curb parking should have enough transverse width to support the motor or non-motor vehicles travelling in this width after parking. This measure can compensate for the lost capacity to some extent as well as increase the safety of non-motor vehicles.

(2) The curb parking zone should be set in road segments without traffic congestion. If traffic congestion appears peak hours, the strategy of time limited parking at peak hours should be implemented in curb parking zones. This measure can reduce the influence of curb parking on traffic conflict and improve motor vehicle safety.

(3) The breadth of curb parking should not be set within the range of the sight triangle at the upstream road segment of a pedestrian crosswalk. The stopping sight distance of motor vehicles can be ensured as well as the safety of cross-street pedestrians. At the same time, segregated installations should be set to forbid pedestrians from crossing the road without a crosswalk.

\section{Conclusions}

(1) The influence of curb parking on the capacity of a road segment is significant. This capacity should be calculated using the effective lane width reduction method or gap acceptance model, according to the transverse remaining width at the parking zone. If the average speed of main traffic flow is $30-40 \mathrm{~km} / \mathrm{h}$, for the situation of only combined driving, the capacity values calculated by the model are less about $12 \%$ than the basic capacity of one lane. For the situation of both combined driving and reduction of lane width, this decrement of capacity is about $22 \%$.

(2) Curb parking has adverse influences on operation safety of dynamic traffic. Among them, motor vehicle safety is determined by the severity of traffic conflict caused by curb parking. Non-motor vehicle safety is determined by the remaining lane width at the parking zone. The safety of crossing pedestrians is determined by whether the stopping sight distance of the driver is satisfied or not. The safety setting distance from parking zone to pedestrian crosswalk should be determined by the average travel speed of main traffic flow to a large extent. However, that value is much larger than that recommended in most of the currant design guidelines.

This research selects four typical road segments with curb parking as its investigation location. Although the number of investigation locations is few, the general regularity acquired by theoretical and case analysis can still be referred to by similar studies. The problem of the number of investigation location should be gradually strengthened by further research.
Fig. 10 The traffic density and conflicts in the parking lane and its adjacent lane

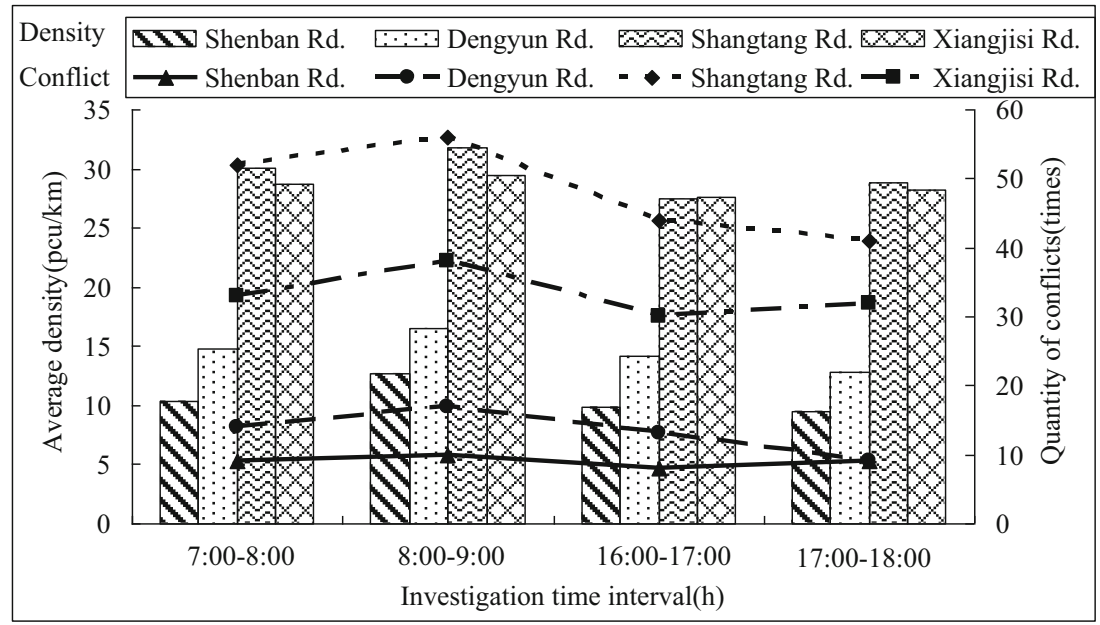


Acknowledgments This research was supported by the Dalian Support Project of Youth Star of Science and Technology under the granted number 2016RQ055. We also acknowledge the editors and anonymous reviewers, for their detailed suggestions, precise comments and continuous helps, which lead to the belief that their dedication contributes to this research and it would not have been feasible without their support.

Open Access This article is distributed under the terms of the Creative Commons Attribution 4.0 International License (http:// creativecommons.org/licenses/by/4.0/), which permits unrestricted use, distribution, and reproduction in any medium, provided you give appropriate credit to the original author(s) and the source, provide a link to the Creative Commons license, and indicate if changes were made.

\section{References}

1. Geng KJ (2013) Necessity and model about supply and demand planning of urban curb parking. Transp Stand 3:42-44

2. Dai S, Liu GJ, Zhu JA, Gong JG, Qu X (2014) On-street parking management strategies and practice. Urban Transp China 12:6-11. doi:10.13813/j.cn11-5141/u.2014.01.007

3. Yousif S, Purnawan (2004) Traffic operations at on-street parking facilities. Proc Inst Civ Eng Transp 157:189-194. doi:10.1680 /tran.157.3.189.41182

4. Jaller M, Holguín J, Hodge S (2013) Parking in the city. Transp Res Rec 2379:46-56. doi:10.3141/2379-06

5. Furth PG, Dulaski DM, Buessing M, Tavakolian P (2010) Parking lane width and bicycle operating space. Transp Res Rec 2190:4550. doi:10.3141/2190-06
6. Edquist J, Rudin CM, Lenné MG (2012) The effects of on-street parking and road environment visual complexity on travel speed and reaction time. Accid Anal Prev 45:759-765. doi:10.1016/j. aap.2011.10.001

7. Wijayaratna S, Wijayaratna K P (2016) Quantifying the impact of on-street parking on road capacity: a case study of Sydney arterial roads. Transportation Research Board 95th Annual Meeting. Washington DC, United States, 10th-14th Jan. 2016, Paper \#16-4405

8. He YQ, Li J (2012) Study on road capacity based on roadside parking. J Civ Eng Manag 29:44-47

9. Liu XM, Wang L (2012) Cellular automation traffic flow model considering on-street parking. J Jilin University 42:327-333. doi:10.13229/j.cnki.jdxbgxb2012.02.036

10. Guo HW, Gao ZY, Zhao XM, Yang XB (2011) Traffic behavior analysis of non-motorized vehicle under influence of curb parking. J Transp Syst Eng Inf Technol 11:79-84. doi:10.16097/j.cnki.10096744.2011.01.010

11. Mei ZY, Chen J, Wang W (2009) A model of road traffic delay caused by curb parking. J Harbin Inst Technol 41:164-168

12. Mei Z Y, Chen J (2012) Modified motor vehicles travel speed models on the basis of curb parking setting under mixed traffic flow. Math Probl Eng. paper No. 351901. doi: $10.1155 / 2012 / 351901$

13. Chen J, Wang Z (2013) Model of bicycle compression wave based on the effect caused by curb parking on bicycle lane. J Harbin Inst Technol 45:114-118

14. Li J, Li JQ, Li J (2010) Research on municipal road width calculation modeling. West China Commun Sci Technol 37:7-11. doi:10.13282/j.cnki.wcest.2010.08.001

15. Transportation Research Board (2010) Highway Capacity Manual (HCM 2010). ISBN 978-0-309-16078-0[Volume 1] 